\title{
QoE Power-Efficient Multimedia Delivery Method for LTE-A
}

\author{
M. Sajid Mushtaq, Abdelhamid Mellouk, Brice Augustin and Scott Fowler
}

\author{
Linköping University Post Print
}

\section{Tweet}

N.B.: When citing this work, cite the original article.

M. Sajid Mushtaq, Abdelhamid Mellouk, Brice Augustin and Scott Fowler, QoE PowerEfficient Multimedia Delivery Method for LTE-A, 2015, IEEE Systems Journal, 1-12. http://dx.doi.org/10.1109/JSYST.2015.2435994

(C)2015 IEEE. Personal use of this material is permitted. However, permission to reprint/republish this material for advertising or promotional purposes or for creating new collective works for resale or redistribution to servers or lists, or to reuse any copyrighted component of this work in other works must be obtained from the IEEE.

\section{http://ieeexplore.ieee.org/}

Postprint available at: Linköping University Electronic Press

http://urn.kb.se/resolve?urn=urn:nbn:se:liu:diva-117941 


\title{
QoE Power-Efficient Multimedia Delivery Method for LTE-A
}

\author{
M. Sajid Mushtaq*, Abdelhamid Mellołk*, Brice Augustin* and Scott Fowler ${ }^{\dagger}$ \\ ${ }^{*}$ LiSSi/TINC Laboratory, Department of Networks and Telecoms, University of Paris-Est Créteil VdM (UPEC), France \\ ${ }^{\dagger}$ Mobile Telecommunications, Department of Science and Technology, Linköping University, Norrköping, Sweden
}

\begin{abstract}
The fastest growing of multimedia services over future wireless communication system demand more network resources, efficient delivery of multimedia service with high users satisfaction, and power optimization of User Equipments (UEs). The resources and power optimization are significant in future mobile computing systems, because emerging multimedia services consume more resources and power. The 4G standard of LTE-A wireless system has adopted the Discontinuous Reception (DRX) method to extend and optimize the UE battery life, while there is no standard scheduling method to distribute the radio resources among the UE. This paper presents a downlink scheduler, i.e. Quality of Experience (QoE) Power Efficient Method (QEPEM) for LTE-A, which efficiently allocates the radio resources and optimizes the use of $U E$ power using the DRX mechanism. We investigates how the different duration of DRX Light and Deep Sleep cycle influences the QoS and QoE of end users, using VoIP over the LTE-A. The QEPEM is evaluated with the traditional methods, in terms of System Throughput, Fairness Index, Packet Loss Rate, and Packet Delay. The QEPEM measures the user's QoE, and feedback to eNodeB for scheduling decisions along with other important parameters. Our proposed method reduces the packet delay, packet loss, and increases the fairness and UE's power saving with high users satisfaction.
\end{abstract}

Keywords: QoE; QoS; LTE; Power Saving; Scheduling; VoIP; DRX; Light Sleep; Deep Sleep; Multimedia.

\section{INTRODUCTION}

The explosion of powerful mobile devices in consumer electronic market, along with the improved capabilities have led to a vast growth of new multimedia services (e.g. Skype, GTalk, Facebook, online gaming, etc.) for web, video, and voice traffic. In parallel, the 3rd Generation Partnership Project (3GPP) has introduced the new radio access technology, LTE and LTE-A (henceforth referred as LTE) that provides high bandwidth to satisfy the UEs with acceptable QoS as compare to $3 \mathrm{G}$ networks; and working on future mobile systems $(5 \mathrm{G})$ to provide more freedom in terms of capacity, connectivity, support diverse set of services, application and UEs along with efficient power utilization. The smart mobile devices support a lot of diverse data applications that cause the frequent use of LTE networks [9].

Initially, 3GPP improves LTE wireless system by considering the important performance parameters, such as high capacity, lower latencies and offering emerging multimedia service (e.g. VoIP, HD video streaming, multi-player interactive gaming and real-time video). It is necessary to manage these performance parameters in an efficient manner. A key performance parameter on the UE electronics device is power, because emerging multimedia services require computationally complex circuitry that drains the UE battery power quickly, as data transmission bandwidth is limited by the battery capacity [31].

The main challenge in any wireless system is to optimize the power consumption at the UE. The DRX method is not a novel approach in LTE [29], because the existing cellular communication systems (e.g. GSM, UMTS) use it to optimize the power consumption at the UE. In Universal Mobile Telecommunications System (UMTS), the DRX method uses two cycles, i.e. Inactivity for UE wakeup and DRX cycle for sleep. The main difference between LTE and early DRX method is that the UE can switch to the sleep state even if the traffic buffer is not empty [16]. In LTE; the DRX states (e.g. Inactivity) depend on the scheduling, because it increases the UE's active time by reinitializing the DRX Inactive timer. The idea is to optimize the UEs battery life, so that it does not run out of power too quickly.

To save power at the UE, the LTE specification uses the DRX method along with Light Sleep and Deep Sleep methods. In DRX Light Sleep method, the UE enters into sleep mode for a shorter period of time. The UE consumes less power in the method than in normal active operational mode, because UE does not switch-off its receiver completely. Meantime, UE's receiver switches between active and sleep mode periodically to receive the scheduled packets. In a case, when the UE does not receive the packet for a long period the UE goes into the DRX Deep Sleep mode, and turned off its receiver completely. The DRX Deep Sleep mode has longer duration than the DRX Light Sleep mode, and does not consume any power. The multimedia traffic directly influences by DRX Sleep mode, because more the increased power saving will result in more packet delays or packet loss. Thus it is required to optimize the DRX parameters for maximum power saving without degrading network performance that directly influences the service quality experienced by the user, especially for realtime multimedia services (e.g. VoIP, video streaming). Quality of Experience (QoE) is not only to consider and evaluate the network quality, but it also estimates the perceived quality of services by users. In this context, our proposed scheduling method plays an important role that considers the DRX parameters in its scheduling decision for best network performance and maximum user's QoE. Quality of Experience (QoE) is a new concept that evaluate the quality of service by considering 
the users' perception.

In this paper, we propose a downlink scheduling method for LTE networks, that calculate the priorities of the UEs by using an opportunistic approach, based on user's QoE and other important parameters for assigning the radio resources among UEs. The priorities of UEs are calculated by considering the following six parameters: user's QoE (i.e. Mean Opinion Score 'MOS'), channel condition, average throughput, UE buffer status, UE DRX status, and GBR/non-GBR traffic. Two traditional scheduling methods are used to evaluate the performance of proposed method, which are Proportional Fair (PF), and Best Channel Quality Indicator (BCQI). The performance assessment is done for delay sensitive VoIP traffic, and impact of power saving on network performance (QoS) and user perception $(\mathrm{QoE})$ is evaluated with the help of LTE System Level simulator from [17].

The paper is structured as follows. Section II contains the related works. Section III discusses the DRX mechanism, while QoE described in Section IV. Section V discusses about E-model. The proposed QEPEM method is presented in Section VI. Section VII, describes the simulation setup, and Section VIII presents the simulation. We conclude the paper in Section IX.

\section{RELATED WORK}

\section{A. Scheduling Schemes}

Scheduling is a process of allocating the physical radio resources among users so as to fulfil the QoS requirement of the multimedia services. A wireless channel has a time-varying behaviour in comparison to wired networks, and as a result both networks have different resource scheduling schemes. The aim of scheduling scheme is to maximize the overall system throughput while keeping fairness, delay and packet loss rate within QoS requirements to satisfy the end users quality experience.

Generally, users are classified based on their traffic characteristics into real time and non-real time. For real time traffic (e.g. video, VoIP and gaming), scheduling must guarantee that QoS requirements are satisfied. The packet loss rate and delay play a vital role in shaping the user experience. The real time traffic packet must arrive to the user within the certain delay threshold otherwise the packet is considered lost or discarded. The scheduling decisions can be made by considering the following parameters: QoS parameters, User's QoE, Channel Quality Indicator (CQI), resource allocation history, buffer status both at the eNodeB and UE.

The Best Channel Quality Indicator (BCQI) scheme assigns radio resources only to those UEs which have reported the best channel conditions in the uplink through the CQI feedbacks to the corresponding eNodeB. In the meantime, those UEs which suffering bad channel conditions will never get radio resources [30]. In the result, the BCQI performs well in terms of throughput but poor in terms of fairness among the UEs.

The Round Robin (RR) method was developed to overcome the shortcoming of fariness of BCQI. It distributes radio resources equally among the UEs to gain high fairness, but the overall system throughput is degraded because it does not consider the channel condition of the UEs. The Proportional Fair (PF) method is used to handle the constraints of high throughput and fairness, as it is based on the trade-off between maximum achievable average throughputs and fairness.

In [22], the authors proposed scheduling method that allocates the resources to UE by considering the three important parameters, which are CQI, UE's buffer length, and traffic types either Real Time (RT) and Non-Real Time (NRT). This method neglects the packet delay factor that can increase the packet loss rate.

In [2], the authors proposed the two layers scheduling method that gave fairness of radio resources and high throughput. However, this method does not consider the packet delay and Guaranteed Bit Rate (GBR) parameters that have a high influence on QoS performance, and user's QoE. Moreover, it does not consider the power saving method which is an important part of LTE networks.

The method proposed in [7] used a time and frequency domain scheduling that maximizes the throughput. The method also make sure that user's delay never exceeds a threshold value and it gets at least minimum throughput to fulfil the QoS requirement. This method fulfils the QoS requirement, but it does not consider the channel condition when allocating the radio resources to UEs.

In [23], the authors proposed a cross-layer resource allocation method, and it evaluated with two scheduling methods, which are PF and Modified Largest Weighted Delay First(MLWDF) with fixed power allocation, and consider the system throughput as a performance metric. The CDF of the normalized user throughput is used to compare the fairness of the proposed method with MAX C/I, RR, and PF. The proposed method does not take into account the packet delay, GBR and other QoS parameters of the LTE networks.

\section{B. DRX Power Saving Method}

The increasing demand of high speed data service, and dramatic expansion of network infrastructure, trigger an enormous increase of energy consumption in wireless network. Today, the optimal energy consumption has become a major challenge, and different methods are proposed for efficient use of power energy of the different elements in wireless network infrastructure.

The DRX power saving method is used in different wireless communication systems with the main purpose to prolong the battery life through monitoring the UE activities. It is based on simple procedure, when there is not any transmitted data, it saves the power by switching-off the UE wireless transceiver. During the sleep state of the UE, the DRX method considerably increases the packet delay.

The DRX mechanism of UMTS is investigated in [34] with the help of an analytical model, where only DRX functionality consists of two parameters; Inactivity Time and the DRX cycle, between the NodeB and UE for saving the power of the UE. The effects of DRX cycles are observed by considering the timers, queue length and packet waiting times. In [36], 
the authors present an analytical model, which prove the LTE DRX mechanism has the ability to save more power than UMTS [28] DRX method.

The power saving methods for two different WiMAX standards, IEEE 802.16e and IEEE $802.16 \mathrm{~m}$ are discussed in [4]. In this paper survey, the authors highlight the important issues related to power saving mechanism in WiMAX networks and address the several problems to improve its efficiency.

The influence of Transmission Time Interval (TTI) sizes, including the the effects of LTE DRX Light and Deep Sleep mode on power consumption are evaluated in [15] for Voice and Web traffic. This study work does not consider the impact of these parameters on QoS. In [3] the DRX-aware scheduling is proposed which includes the DRX status as a scheduling decision parameter to reduce packet delay caused by the DRX sleep duration. The scheduling priority is directly proportional to delay of a head of line packet delay in relation to the remaining active time before a UE enters into sleep mode. In [11] semi-persistent scheduling scheme for VoIP is developed using the DRX. First it organizes the UEs into the scheduling candidate set (SCS) based on the UE buffer information at the eNodeB, the DRX status and the persistent resource allocation pattern. It calculates the priority metric for the UEs in SCS by favoring the UEs who require retransmissions then the UEs whose packet delay of unsent packet in the eNodeB buffer is close to delay threshold. Both schemes presented in [3] and [11] use DRX mechanism to optimize power usage and offer solutions to the problems caused by the sleep interval of increased packet delay and packet loss. However, both schedulers do not consider GBR requirement of UEs.

In [1], the performance of DRX mechanism is evaluated in terms of DRX cycle lengths and related timer values, by observing their effect on VoIP traffic service over the High Speed Downlink Packet Access (HSDPA) network. However, the battery life of UE might a key limiting factor in providing satisfactory user experience. The results showed that longer DRX cycle saves more UE power but at the same time VoIP capacity over HSDPA can be compromised in the case when there are not suitable selection of DRX parameters are applied.

In [35], the authors present the semi-Markov chain model to analysis the impact of DRX mechanism in LTE network with Machine Type Communication (MTC) traffic, while in [21], the authors proposed the method for modelling the DRX mechanism in LTE wireless networks with the help of Poisson traffic. In the same way, in [16], the analytical model is used to study the influence of fixed and adjustable DRX cycle mechanism in LTE network, using the bursty packet data traffic with the help of semi Markov process. However, these proposed methods [35], [21], and [16], do not consider the QoS features such as fair resources allocation, packet loss rate and throughput, which are badly effected with the DRX mechanism in LTE networks.

The impact of LTE DRX Light Sleep mechanism on QoS is examined in [25], using the VoIP traffic model. However, the performance is evaluated only with the LTE DRX Light Sleep Cycle, and Deep Sleep Cycle was not considered. In [20], the DRX optimization is performed for the mobile internet application by considering the DRX inactivity timer and the DRX cycle length with two users. This method is evaluated with only two users, and it also does not take into account the impact on other QoS parameters like fairness, throughput, packet loss rate, and GBR requirement for RT traffic. Our proposed QEPEM method takes scheduling decision based on six parameters (user's QoE, channel condition, average throughput, UE buffer status, UE DRX status, and GBR/nonGBR traffic) with DRX Light Sleep Cycle and Deep Sleep Cycle. We have individually observed the influence of each DRX Light Sleep and Deep Sleep Cycle on 15 UEs in the context of Throughput, Fairness, Delay and Packet Loss Rate.

\section{DRX MECHANISM}

The DRX mechanism has been implemented on 2G (GSM) and 3G (UMTS) cellular networks. LTE specification has adopted DRX at the link level to save power and extend battery life of the UE. In LTE networks, the DRX mechanism can observe the Radio Resource Control (RRC) states between the UEs and eNodeB [31]. The RRC has two different states where DRX mechanism can be worked, i.e., RRC_Idle and RRC_Connected.

In the RRC_Idle state, the UE is registered in the LTE network with specific unique identifier, but it does not has active session with the eNodeB. In this state, the eNodeB can page the UE at any time for a different purpose (e.g., get location information), while the UE can request an uplink channel by establishing a $R R C$ Connected state, so that it can receive and transmit data. In the $R R C$ _Connected state, the DRX mode can be enabled during idle periods between successive packet arrivals. In case there is no data packet the UE can go into DRX mode.

The LTE's DRX mechanism, the sleep/wakeup scheduling of each UE receiver could be described in terms of three periods (ON-Duration, Inactivity and Sleep Interval) as shown in Figure 1. The values of LTE's DRX parameter are defined in [31]. In this paper, we are considering the following parameters:

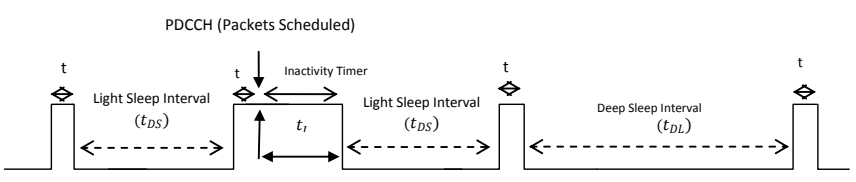

Figure 1: LTE DRX Mechanism at UE

- DRX cycle: It is a time interval between the start of two consecutive ON-Duration in which UE remains active. One DRX cycle consists of an ON-Duration and a Sleep interval.

- ON-Duration $(t)$ : It is the time when the UE is in active state, and listening to the PDCCH. If any data packet is scheduled, the UE starts its Inactivity $\operatorname{Timer}\left(t_{I}\right)$; otherwise it continues its DRX Sleep cycle. In this paper, 
we set the value of ON-Duration to $1 \mathrm{~ms}$, because this timer just checks the availability of scheduled data.

- Inactivity Timer $\left(t_{I}\right)$ : When a packet is found during ON-Duration, the UE starts its $t_{I}$ and receives data packets. During $t_{I}$, if another PDCCH packet arrives, the Inactivity time restarts itself timer. When $t_{I}$ expires, DRX cycle starts with a sleep interval. The value of $t_{I}$ is set to $5 \mathrm{~ms}$.

- Sleep Interval: It is a time interval during which the UE is either in DRX Light Sleep $\mathrm{t}_{D S}$ mode (consume low power) or in DRX Deep Sleep $t_{D L}$ (consume no power) mode. In Deep Sleep mode, the sleep interval is longer than Light Sleep mode. This paper considers the values of Light Sleep duration are 2, 5, 10, 16, $20 \mathrm{~ms}$ and for Deep Sleep duration 10, 20 , 42, 64, and $80 \mathrm{~ms}$ [31], as longer duration saved more power, but also increased the packet delay.

We can use a semi-Markovian model to determine the numerical values of power saved in DRX mechanism. In [6], a semi-Markovian model is presented to save UE power for LTE networks as shown in Figure 2, which is used by [36] and [15]. This model shows that when the UE in the active state downloading the data, it consumes 0.5 Watt/TTI. However, if the UE in Light Sleep mode, it consumes 0.011 Watt/TTI, that means it saves $0.489 \mathrm{Watt} / \mathrm{TTI}$. On the other hand, in the case of the Deep Sleep mode the UE does not utilize any power (i.e. $0 \mathrm{Watt} / \mathrm{TTI})$ that represents the full power saving mode.



Figure 2: Semi-Markovian Model for Power Consumption

\section{QUALITY OF EXPERIENCE FOR VOIP}

Voice over IP (VoIP) is a popular low cost service for voice calls over IP networks. The success of VoIP is mainly influenced by user satisfaction, in the context of quality of calls as compared to conventional fixed telephone services. Initially, the implementation of VoIP services was unable to handle the unpredictable behaviour of IP networks, which badly affected the growth of early VoIP services, because its traffic streams are both delay and loss sensitive. It is a main challenge for VoIP services to provide the same QoS as a conventional telephone network, i.e. reliable and with a QoS guarantee.

In conventional networks, the 'Bearer' quality is managed as a single quality plan, while in Next Generation Networks (NGNs), it is also necessary to manage end-users' QoE. In a wireless system, the unpredictable air interface behaves differently for each UE. In these circumstances, it is necessary to monitor the QoE in the network on a call-by-call basis [27].

Quality of Experience (QoE) is a new concept that evaluate the quality of service by considering the users' perception.
Many network researchers are now working on this concept, and trying to integrate it in network decisions to ensure a high customer satisfaction with minimum network resources. In this context, our proposed algorithm takes the scheduling decision by considering the user satisfaction factor. Generally, QoE is considered as a subjective measure of user satisfaction of a given service. According to [26], the standard definition of QoE is: a measure of the overall acceptability of an application or service, as perceived subjectively by the end-user.

In fact, two methods can be used to evaluate the quality of multimedia services: the Subjective and the Objective method. Subjective method is proposed by the International Telecommunication Union (ITU-T) Rec. P.800 [14] which is mostly used to find out users' perception of the quality of speech. The Mean Opinion Score (MOS) is an example of a subjective measurement method in which users rate the voice quality by giving five different point score from 5 to 1 , where 5 is the best and 1 is the worst quality. However, Objective method uses different models of human expectations and tries to estimate the performance of speech service in an automated manner, without human intervention. It is very difficult to measure subjectively the MOS of inservice speech quality because MOS is a numerical average value of a large number of user's opinion. Therefore, some Objective speech quality measurement methods are developed to make a good estimation of MOS. The E-model [24] and Perception Evaluation of Speech Quality (PESQ) [10] are Objective methods for measuring the MOS scores. The PESQ cannot be used to monitor the QoE for real-time calls, because it uses a reference signal and compares it to the real degraded signal to calculate the MOS score. Therefore, we have used the E-model computational method to calculate the MOS score of conversation quality by using the latency (delay) and packet loss rate with the help of the transmission rating factor (Rfactor) [24].

\section{E-MODEL}

The E-model defined in the ITU-T Rec. G. 107 [24], is an analytical model of voice quality, which is used for the network planning purposes. In the E-model, the basic result is to calculate the $R$ - factor, that measures the voice quality ranging from 100 to 0 , where 100 is the best and 0 is the worst quality. The R-factor value is used to determine the MOS value, which is the arithmetic average of user opinion. The MOS value is obtained from $R$-factor by using the equation (1) [33].

$$
M O S= \begin{cases}1 & R<1 \\ 1+0.035 R+R(R-60)(100-R) 7 \cdot 10^{-6} & 0<R<100 \\ 4.5 & R>100\end{cases}
$$

The general correlation between $R$-factor, MOS scores and the quality of user experience with the VoIP service is shown in Table I. The high value of $R$-factor gives the highest MOS score, and the user gets the best QoS with high satisfactory experience. 
Table I: Correlation between R-Factor, MOS and User's Experience

\begin{tabular}{|c|c|c|}
\hline $\begin{array}{c}\text { R-Factor } \\
\text { (lower limit) }\end{array}$ & $\begin{array}{c}\text { MOS } \\
\text { (lower limit) }\end{array}$ & User Experience \\
\hline 90 & 4.34 & Excellent \\
\hline 80 & 4.03 & Good \\
\hline 70 & 3.60 & Fair \\
\hline 60 & 3.10 & Poor \\
\hline 50 & 2.58 & Bad \\
\hline
\end{tabular}

The $R$ - factor mainly depends on four parameters as shown in equation (2)

$$
R=R_{o}-I_{s}-I_{d}-I_{e f}+A
$$

where $R_{O}$ represents the basic signal-to-noise ratio, which includes noise sources such as circuit and room noise, $I_{s}$ is a combination of all impairments with voice signal, $I_{d}$ is the impairment's factor caused by delay, $I_{e f}$ is an effective equipment impairment factor associated with the losses as it is defined in [12], and $A$ is the advantage factor. In [18], ITU-T provides the common values of impairment factors. After selecting the default values, we can obtain the reduced expression for the $R$ - factor in equation (3).

$$
R=94.2-I_{d}-I_{e f}
$$

Equation (3) clearly shows that $R$-factor mainly depends on the end-to-end delay and total loss probability, which affect the VoIP call quality. The delay components $\left(I_{d}\right)$ is provided in [24] and its influence on voice quality depends on a critical time value of $177.3 \mathrm{~ms}$, which is the total delay budget for VoIP streams. The impact of this delay is modelled in [5], and it is given in equation (4)

$$
I_{d}=0.024 d+0.11(d-177.3) H(d-177.3)
$$

where $d$ is the one way delay (in milliseconds) and $H(x)$ is a step function as mentioned in equation (5)

$$
H(x)= \begin{cases}0 & \text { if } \mathrm{x}<0 \\ 1 & \text { if } \mathrm{x} \geq 0\end{cases}
$$

The quality of a VoIP call also depends on loss impairment $\left(I_{e f}\right)$, as it is clearly shown in equation (3). In order to find the expression for calculating the value of $I_{e f}$, we use the methods as proposed in [5], [8] and [32] that consider the overall packet loss rate as

$$
I_{e f}=\gamma_{1}+\gamma_{2} \ln \left(1+\gamma_{3} e\right)
$$

where $e$ is the total loss probability (including network and buffer) which has a value between 0 and $1, \gamma_{1}$ represent the voice quality impairment factor caused by the encoder, while $\gamma_{2}$ and $\gamma_{3}$ represent the impact of loss on voice quality for a given codec. In case of a G.729-A codec, $\gamma_{1}=11, \gamma_{2}=40$ and $\gamma_{3}=10$, while for a G.711 codec, $\gamma_{1}=0, \gamma_{2}=30$ and $\gamma_{3}=15$ as presented in [5]. The final expression of $R$-factor by using the G.729-A codec is given in equation (7)

$R=94.2-0.024 d-0.11(d-177.3) H(d-177.3)-11-40 \ln (1+10 e)$

\section{PRoposed QEPEM Method}

The user's QoE is significantly influenced by the QoS parameters. However, there is always a trade-off between the QoS and power saving, because power saving mechanism badly effected the QoS such as delay. In this context, a new downlink scheduling method is proposed that efficiently utilizes the power, and keep balance between QoS and power consumption, while also consider their impact on the user's QoE. The proposed QoE Power Efficient Method (QEPEM) uses an opportunistic scheduling approach that calculates the priorities of UEs and assigns resources to them. This opportunistic scheduling approach is based on the six important scheduling dependencies that have greater impact on QoS and Power saving mechanism, which are MOS, Channel Quality Indicator (CQI), Average throughput history, UE buffer, GBR/non-GBR traffic, DRX status. The MOS score is calculated from $R$ - factor which considers all types of delay (network, buffer, and codec) and packet loss (network and UE's playout) factors, as a result the MOS score represents the overall effect of delay and packet loss. The priority for each Resource Block (RB) is estimated for every UE; the scheduler assigns RB to a UE whose priority value is the highest amongst all other UEs for that specific RB.

To calculate the priorities, the algorithm first estimates maximum achievable throughputs for every RB if assigned to UEs according to channel conditions reported by UEs. In order to balance between system throughput and fair resource distribution the proposed scheduler (henceforth is referred to as QEPEM) utilizes the property of Proportional Fair (PF) which is defined in [30].

$$
\begin{gathered}
\text { fair_factor }_{i}=\frac{\text { achievable_throughput }_{i j}}{\text { average_throughput }_{i}} \\
R_{i}(t)=\left(1-\frac{1}{t_{c}}\right) * R_{i}(t-1)+\frac{1}{t_{c}} * r_{i}(t-1)
\end{gathered}
$$

In Equation (8), achievable_throughput $t_{i j}$ represents a theoretical achievable throughput of $R B_{j}$ if assigned to $U E_{i}$ at Transmission Time Interval (TTI). In Equation (9), $R_{i}$ represents the average_throughput $t_{i}$ of $U E_{i}$ over a window $t_{c}$ at every TTI and $r_{i}$ is achievable throughput of $U E_{i}$. The window size $t_{c}$ is an important element which is used to calculate the average data rate experienced by each UE.

The priority function $P_{i j}$ calculates priorities of Non RealTime $(N R T)$ and RealTime $(R T)$ services from Equations (10) and (11), respectively. In this study, $R T$ VoIP is used to evaluate the proposed QEPEM method, while how to calculate the user's perception (MOS) for different $N R T$ traffic can be considered in future work. 


$$
\begin{gathered}
P_{i j}=M_{i} * \delta_{i}\left(\text { fair_factor }_{i}\right), \quad i \text { is NRT UE (10) } \\
P_{i j}=M_{i} * \delta_{i}\left(\text { fair_factor }_{i}\left(\frac{\text { GBR }}{\text { average_throughput }}\right)^{\varnothing}\right), \\
i \text { is RT UE }
\end{gathered}
$$

where $\varnothing$ is a tunable exponential factor for GBR and $\delta$ is a DRX status indicator for each UE. The $P_{i j}$ is a priority matrix for each $R B_{j}$ if assigned to $U E_{i}$ while fair_factor f $_{i}$ in accordance to equations (8). GBR is the guaranteed bit rate requirement for GBR UEs. The tunable exponential factor $\varnothing$ can be used to adjust preferences of GBR UEs; if a UE would achieve lower than the average throughput required by the GBR, the scheduler will increase the priority of that UE to fulfil the GBR requirement and vice versa. The $M O S_{i}$ is a priority multiplier that increases the priority of UEs whose facing the degradation of service due to delay and packet loss rate, as higher priority to prevent packet loss. The GBR are irrelevant for NRT traffic because NRT traffic is not delay sensitive and they do not require minimum data rates to guarantee.

The QEPEM is designed in conjunction with DRX mechanism as to fully exploit high bandwidth efficiency of LTE. The DRX manager at the eNodeB shares DRX status with the UEs. On each TTI, the scheduler must consider only UEs that are in active mode of operation then allocate resources for data transmission; this is achieved by including the DRX status in priority criteria. DRX status $\delta$ defines the state of UE, when a UE is in-active mode $\delta=1$. When a UE is in Sleep mode $\delta=0$ makes that UE out of the scheduling competition. Thus the scheduler helps reducing resource wastage by considering only the UEs that are in active state.

Practically, QEPEM method can implement in LTE-A networks without complexity, as DRX mechanism is already working in $2 \mathrm{G} / 3 \mathrm{G} / 4 \mathrm{G}$ networks. On the other hands, user's QoE is an averaged value that could easily calculate based on QoS parameters (delay, and packet loss rate) and other network impairment factors as mentioned in Section V. The computed user's QoE can feedback to the eNodeB, where it can play a vital role in order to improve the user's QoE by considering it in scheduling decision.

\section{Simulation SETUP}

The simulation setup consists of LTE network that is operating at $2 \mathrm{GHz}$ operating frequency, and $5 \mathrm{MHz}$ system channel bandwidth. The eNodeB is considered to be static, which is serving 15 VoIP traffic UEs that are uniformly distributed within the sector and allowed to move randomly. These UEs can be considered as pedestrians moving with a speed of 5 $\mathrm{km} / \mathrm{h}$. In this paper, we use the VoIP traffic model according to [13]. The VoIP traffic model is considered due to the major usage on the UEs. Additionally, fading models are used to simulate realistic channel conditions. DRX Light and Deep Sleep mechanism are implemented on the UEs for saving power, on the other hand, each UE has a finite buffer length at eNodeB that buffered data when the UE in sleeping mode.

A longer Deep Sleep duration can cause the buffer overflow of UE at the eNodeB, because a number of packets being created would be much higher than packets being scheduled. In this paper the DRX ON-Duration and the In-Active parameters are set to 1 TTI and 5 TTIs, respectively to avoid the UE buffer overflow at eNodeB. The power saving effect on user's QoE is considered in the terms of QoS parameters that will be presented and discussed, which are Average System Throughput, Average Throughput Fairness Index, Packet Loss Rate (PLR) and Average Packet Delay. The three performance evaluation parameters are well known, however, the Fairness Index can be defined in terms of system resource allocation or throughput. Jains equation is used to obtain a throughput fairness index. In [19], fairness index $J$ for $n$ UEs is defined as

$$
J\left(x_{1}, x_{2}, \ldots, x_{n}\right)=\frac{\left(\sum_{i=1}^{n} x_{i}\right)^{2}}{n \sum_{i=1}^{n} x_{i}^{2}}
$$

where $x_{i}$ is the throughput for the $i^{t h}$ UE. The best case can give a maximum value of 1 , which means all UEs achieved exactly the same throughput. When the difference between the UEs throughput increase then the value of Jain's equation decreases.

The important simulation parameters are listed in Table II and the duration of Light and Deep Sleep mode cycle are

\begin{tabular}{|c|c|}
\hline Parameters & Values \\
\hline eNode $B$ radius & $250 \mathrm{~m}$ \\
\hline Number of sectors per eNodeB & 3 \\
\hline Target area & Single sector \\
\hline Number of UEs & 15 \\
\hline eNodeB total TX power & $20 \mathrm{~W}$ \\
\hline Number of antennas (SISO) & $1 \mathrm{TX}, 1 \mathrm{RX}$ \\
\hline Fading models & Fast fading \\
\hline UE Speed & $5 \mathrm{~km} / \mathrm{h}$ \\
\hline Operating frequency band & $2 \mathrm{GHz}$ \\
\hline System channel bandwidth & $5 \mathrm{MHz}$ \\
\hline Number of RBs & 25 \\
\hline$\varnothing$ & 2 \\
\hline GBR & $25 \mathrm{kbps}$ \\
\hline CQI reporting & Every TTI \\
\hline Traffic model & VoIP \\
\hline VoIP packet generation interval & $20 \mathrm{~ms}$ \\
\hline VoIP delay threshold & $100 \mathrm{~ms}$ \\
\hline Power saving mechanism & DRX Light and Deep Sleep \\
\hline DRX on duration & 1 TTI \\
\hline DRX In-Active duration & 5 TTIs \\
\hline DRX Light Sleep duration & $2,5,10,16,20(\mathrm{~ms})$ \\
\hline DRX Deep Sleep duration & $10,20,40,64,80(\mathrm{~ms})$ \\
\hline
\end{tabular}
selected according to 3 GPP TS 36.331 version 8.8.0 Release 8 .

Table II: Main Simulation Parameters

\section{Simulation Results}

The performance of the proposed QEPEM method has been evaluated, and compared with two traditional scheduling 
algorithms; Proportional Fair (PF), and Best CQI (BCQI) in power saving mode. The evaluation and comparison is done with the same simulation environment and parameter.

\section{A. Performance Analysis with Fixed Deep Sleep $20 \mathrm{~ms}$}

The simulation setup are same for all schedulers as given in Table II, and performance are evaluated in the varying power saving environment DRX Light Sleep with fixed Deep Sleep mode of 20TTI (20ms). The DRX mechanism is applied on the UEs along with the fixed DRX ON-Duration of 1 TTI, while the In-Active duration set to 5TTI. The simulation executes for different Light Sleep parameters, but due to space limitation only Figure 3 is given while impact of other parameters are summarized in Table III.

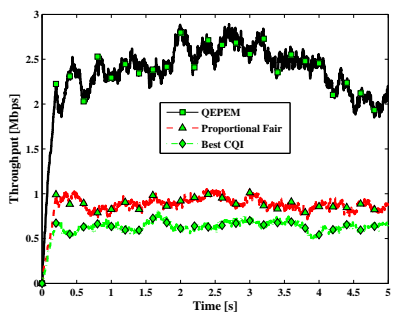

(a) Average Throughput

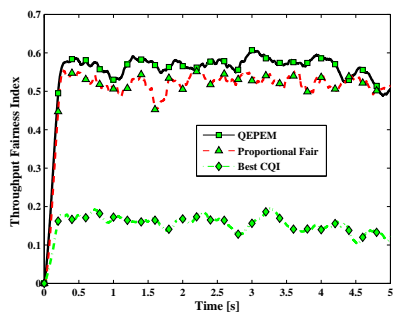

(b) Throughput Fairness Index
Figure 3: Light Sleep $=20 \mathrm{~ms}$, Fixed Deep Sleep $=20 \mathrm{~ms}$

Figure 3a shows the average system throughput when the simulation runs for $5000 \mathrm{TTI}$, which are equal to 5 seconds. The results are obtained, when the duration of DRX Light Sleep Cycle is set to $20 \mathrm{~ms}$ (20 TTI) with a fixed duration of the DRX Deep Sleep Cycle, which is equal to $20 \mathrm{~ms}$ (20 TTI). The result shows that the throughput of the proposed QEPEM method is significantly higher as compared to all other schedulers. QEPEM uses the DRX information of each UE, in other words the QEPEM method considers the ON-Duration and Inactive duration of all UEs during the scheduling decision. The traditional schedulers are designed to consider all UEs that are connected at the time scheduling is performed. PF hold second position in terms of throughput because it also tries to balance the throughput with the resource fairness. BCQI performed the worst in this regard, due to the fact that BCQI chooses only those UEs which have the best channel conditions in the uplink through the CQI feedbacks.

Figure 3b, illustrates the Throughput Fairness Index according to Jains equation. The result clearly shows that proposed QEPEM method performed the best as compared to all other scheduling schemes. The QEPEM manages to achieve higher fairness, because it considers the channel conditions and UEs GBR requirements. It tries to allocate resources to those UEs which packets are residing in the eNodeB buffer for a longer time to avoid the packet lost, and improve the user's QoE. Similarly if the UEs is lacking in throughput according to their defined GBR requirement, then it again allocates more radio resources to those UEs. PF does not consider the sleeping state of UEs, but it tries to achieve fairness among them by considering the performance history of each UE. It follows the pattern of QEPEM method. The value of BCQI is close to worst case scenario as it allocates the resources only to those UEs which report good channel condition.

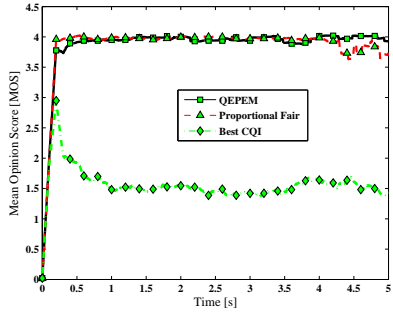

(a) Mean Opinion Score

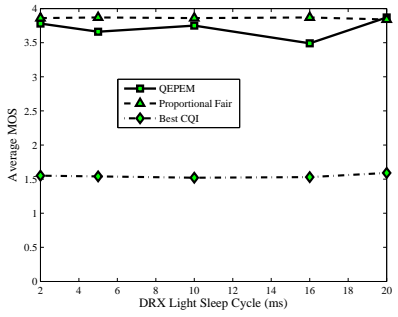

(b) Average MOS Value
Figure 4: Light Sleep $=20 \mathrm{~ms}$, Fixed Deep Sleep $=20 \mathrm{~ms}$

Figure 4 illustrates the performance of three schedulers in terms of user's perceived QoE, when DRX Light Sleep cycle has duration of $20 \mathrm{~ms}$ along with fixed Deep Sleep duration of 20ms. Figure 4a shows that QEPEM and PF have almost the same performance; while BCQI has worst performance. Similarly, Figure $4 \mathrm{~b}$ shows that performance of PF is close to proposed QEPEM method, except when the Light Sleep has duration of $16 \mathrm{~ms}$. BCQI has bad performance, as it deals only the limited UEs that are reporting the same channel quality.

Table III: Schedulers Evaluation, Fixed Deep Sleep 20 ms

\begin{tabular}{|c|c|c|c|c|c|c|}
\hline Light & Scheduler & Throughput & F-Index & Delay & PLR & MOS \\
\hline \multirow{4}{*}{2} & QEPEM & 3.707 & 0.5894 & 9.8714 & 0 & 3.78 \\
\cline { 2 - 7 } & PF & 1.043 & 0.5350 & 17.6295 & 0.00059 & 3.86 \\
\cline { 2 - 7 } & BCQI & 1.566 & 0.1410 & 38.1408 & 0.4675 & 1.55 \\
\hline \multirow{4}{*}{5} & QEPEM & 3.3865 & 0.6001 & 8.9632 & 0 & 3.66 \\
\cline { 2 - 7 } & PF & 1.0586 & 0.5362 & 17.3929 & 0.00043 & 3.87 \\
\cline { 2 - 7 } & BCQI & 1.3412 & 0.1470 & 33.8087 & 0.4494 & 1.55 \\
\hline \multirow{3}{*}{10} & QEPEM & 2.6513 & 0.5393 & 10.5643 & 0.0024 & 3.75 \\
\cline { 2 - 7 } & PF & 1.0316 & 0.5385 & 17.8852 & 0 & 3.86 \\
\cline { 2 - 7 } & BCQI & 1.1071 & 0.1522 & 34.2655 & 0.4600 & 1.52 \\
\hline \multirow{4}{*}{16} & QEPEM & 2.7837 & 0.5646 & 9.1295 & 0.0127 & 3.49 \\
\cline { 2 - 7 } & PF & 0.86255 & 0.4812 & 17.1979 & 0 & 3.87 \\
\cline { 2 - 7 } & BCQI & 0.7513 & 0.1523 & 36.6407 & 0.4634 & 1.53 \\
\hline \multirow{3}{*}{20} & QEPEM & 2.3923 & 0.5617 & 10.7919 & 0.0013 & 3.87 \\
\cline { 2 - 7 } & PF & 0.8861 & 0.5191 & 19.4660 & 0 & 3.84 \\
\cline { 2 - 7 } & BCQI & 0.6382 & 0.1554 & 29.8075 & 0.4568 & 1.59 \\
\hline
\end{tabular}

Table III summarizes the results of different Light Sleep Cycle with fixed Deep Sleep mode of $20 \mathrm{~ms}$. The average values of different performance parameters are given in terms of system throughput, throughput fairness index, packet delay and packet loss rate. The average value of packet delay shows that the QEPEM scheduler achieved the least delay followed by the PF scheduler, which has performed better than the BCQI scheduler. The proposed QEPEM method performs the best as compare to other methods in term of Throughput, Faireness Index, and Delay, while in terms of PLR, QEPEM performs best than BCQI, but sometime its performance close to PF. The BCQI scheduler performed the worst in all cases, as it assigns radio resources to the limited UEs. 


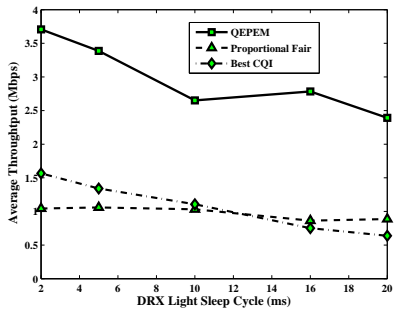

(a) Average Throughput

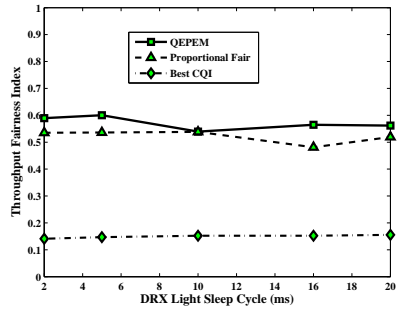

(b) Fairness Index
Figure 5: Vary Light Sleep with Fixed Deep Sleep $=20 \mathrm{~ms}$

Figure 5, shows the Average Throughput and Fairness Index for three scheduling method QEPEM, PF, and BCQI. The results show the impact of DRX Light Sleep duration along with fixed Deep Sleep duration equal to $20 \mathrm{~ms}$. Figure 5a, depicts that the QEPEM performs better due to the fact that it is designed to provide better fairness among the UEs by fulfilling the GBR UEs requirements at the cost of lower system throughput. The results clearly represent that QEPEM is least affected by increase in sleep durations because it considers the DRX state of the UEs and user perception in order to maximize QoE. BCQI and PF scheduler performance degraded significantly when the system is working in power saving mode. The figure clearly shows that QEPEM is performing better than the other schemes if the duration of DRX sleep is increased. Figure 5b shows that QEPEM performs better than other methods, while the performance of PF is close to proposed QEPEM. BCQI performed the worst best in this case due to its resource distribution policy.

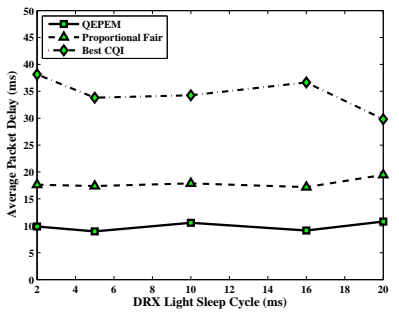

(a) Average Packet Delay

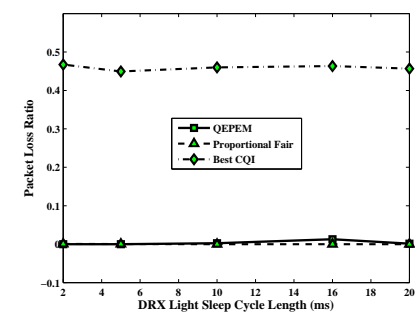

(b) Average Packet Loss Rate
Figure 6: Vary Light Sleep with Fixed Deep Sleep $=20 \mathrm{~ms}$

Figure 6 illustrates the effect of power saving on packet delay shown in Figure 6a, and packet loss rate presented in Figure $6 \mathrm{~b}$ for three scheduling methods. In case of VoIP Communication, it is required that when a packet is created, it must reach the UE within $100 \mathrm{~ms}$ as per QCI characteristic of LTE networks; otherwise the packet will be discarded. It is observed that when the DRX Light Sleep duration increases, the packets start to suffer more delay, because the packet delay is directly proportional to the power being saved through the DRX sleep duration. Figure 6, depicts that QEPEM performed the best, and PF method came second in terms of packet delay and packet loss rate. The results show that both of these scheduler follows the linear pattern. QEPEM Scheme is designed to reduce the packet delays and losses while achieving the high throughput and fairness to improve the user's QoE. BCQI performs worst in terms of packet delay and packet loss rate, because it is designed to achieve maximum system throughput in normal operational mode, yet it disregards fairness and delay constraints.

\section{B. Performance Analysis with Fixed Light Sleep $10 \mathrm{~ms}$}

The impact of power saving mechanism on user's QoE and QoS in the LTE networks will be evaluated by fixing the DRX Light Sleep Cycle to $10 \mathrm{~ms}$ and observed the effect of different DRX Deep Sleep Cycle duration. The impact of each Deep Sleep duration is evaluated, while the results are summarized in Table IV due to space limitation.

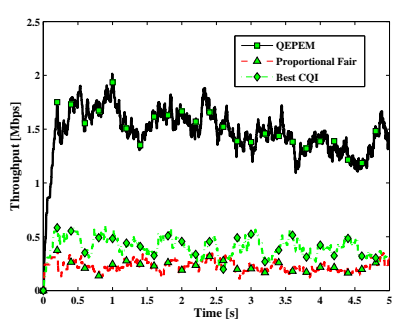

(a) Average Throughput

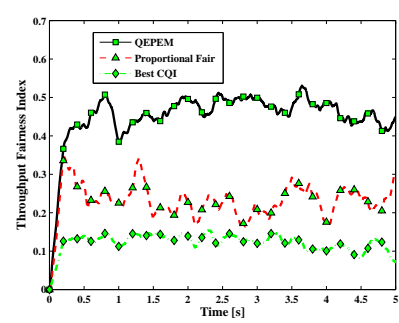

(b) Throughput Fairness Index
Figure 7: Deep Sleep $=80 \mathrm{~ms}$, Fixed Light Sleep $=10 \mathrm{~ms}$

Figure 7 depicts the Average throughput and fariness index, when the DRX Light Sleep Cycle has a value of $10 \mathrm{~ms}$ with a DRX Deep Sleep Cycle duration set to 80ms. QEPEM has the best performance in terms of throughput and fairness, due to its efficiency of scheduling decision which is based on important parameters (e.g. DRX, MOS, GBR, etc.). In addition QEPEM, $\mathrm{PF}$ is performing better in contrast to traditional BCQI scheme. By increasing the duration of the Deep Sleep cycle, the average throughput of all the scheduling schemes reduced. Figure $7 \mathrm{a}$ shows that QEPEM again achieves the highest throughput than traditional schedulers, because it assigns the resources to those UEs that are in-active mode, which results to achieve high fairness index as shown in Figure $7 \mathrm{~b}$.

Figure 8 depicts the user's perceived QoE in the form of MOS values while using the three scheduling methods, when the DRX Deep Sleep Cycle has a value of $80 \mathrm{~ms}$ with a fixed DRX Light Sleep Cycle duration set to $10 \mathrm{~ms}$. Figure 8a, clearly shows that the proposed QEPEM has achieved the high user's satisfaction along with a large power saving at the UE. This is because the proposed QEPEM method considers the user's perception and DRX status while making the scheduling decision. BCQI holds the second position, while PF has the worst performance in this case scenario. Figure $8 \mathrm{~b}$ represents the performance of three scheduling method using the Average MOS performance metric. It is observed that when the duration 


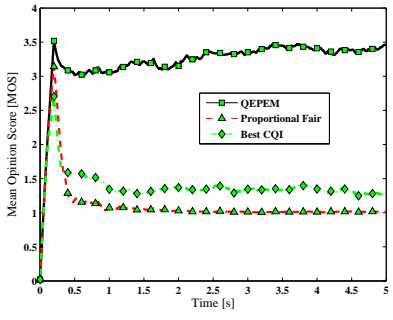

(a) Mean Opinion Score

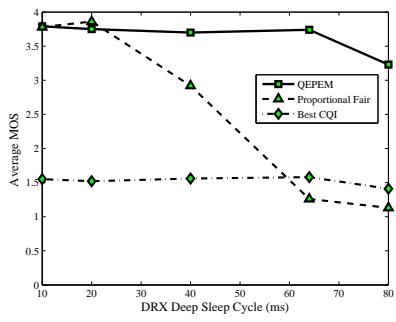

(b) Average MOS Value

Figure 8: Deep Sleep $=80 \mathrm{~ms}$, Fixed Light Sleep $=10 \mathrm{~ms}$

of the Deep Sleep cycle is increased then Average MOS of PF is significantly reduced. QEPEM method again achieves the highest user's satisfaction as compared to the other traditional methods. BCQI has almost the same behaviour as it servers only the limited UEs that face the same network quality.

Table IV: Schedulers Evaluation, Fixed Light Sleep 10 ms

\begin{tabular}{|c|c|c|c|c|c|c|}
\hline Deep & Scheduler & Throughput & F-Index & Delay & PLR & MOS \\
\hline \multirow{4}{*}{10} & QEPEM & 3.5172 & 0.5838 & 5.7893 & 0 & 3.79 \\
\cline { 2 - 7 } & PF & 1.3473 & 0.5643 & 8.6391 & 0 & 3.78 \\
\cline { 2 - 7 } & BCQI & 1.2000 & 0.1549 & 32.2102 & 0.4103 & 1.55 \\
\hline \multirow{4}{*}{20} & QEPEM & 2.6513 & 0.5393 & 10.5643 & 0.0024 & 3.75 \\
\cline { 2 - 7 } & PF & 1.0316 & 0.5385 & 17.8852 & 0 & 3.86 \\
\cline { 2 - 7 } & BCQI & 1.1071 & 0.1522 & 34.2655 & 0.4600 & 1.52 \\
\hline \multirow{4}{*}{40} & QEPEM & 2.2174 & 0.5178 & 19.9674 & 0.0098 & 3.70 \\
\cline { 2 - 7 } & PF & 0.52386 & 0.4211 & 38.2932 & 0.0517 & 2.92 \\
\cline { 2 - 7 } & BCQI & 0.74179 & 0.1431 & 42.2722 & 0.5346 & 1.56 \\
\hline \multirow{3}{*}{64} & QEPEM & 1.9751 & 0.4815 & 30.1797 & 0.0125 & 3.47 \\
\cline { 2 - 7 } & PF & 0.30250 & 0.2918 & 49.9616 & 0.3565 & 1.26 \\
\cline { 2 - 7 } & BCQI & 0.65989 & 0.1255 & 48.7677 & 0.6073 & 1.58 \\
\hline \multirow{3}{*}{80} & QEPEM & 1.5037 & 0.4605 & 37.0972 & 0.0250 & 3.23 \\
\cline { 2 - 7 } & PF & 0.23113 & 0.2369 & 53.2352 & 0.4865 & 1.13 \\
\cline { 2 - 7 } & BCQI & 0.409880 & 0.1239 & 57.9616 & 0.6298 & 1.41 \\
\hline
\end{tabular}

Table IV sums up the performance of three schedulers QEPEM, PF, and BCQI in the forms of four important QoS parameters (throughput, fairness index, packet delay, and packet loss rate) that have high influence on the user's perceived QoE. When the duration of Deep Sleep Cycle increases, the performance of all schedulers are degraded. However, the proposed QEPEM has successfully manages this situation by considering the DRX and user's perception in its scheduling decision. QEPEM has the high system throughput, fairness index, and least packet delay in comparison to the others schedulers, while in case of PLR, the QEPEM has also better performance than $\mathrm{PF}$ except when the Light Sleep has value $20 \mathrm{~ms}$, where QEPEM performance is very close to PF. BCQI has the worst performance in all scenarios, because it allocates the resources to fewer UEs by considering the channel quality.

Figure 9 illustrates the performance of QEPEM, PF, and BCQI in terms of QoS parameters, which are average throughput and fairness index. The system throughput is averaged over 5000 TTIs for each scheduler. The QEPEM performs better as compared to the other traditional schemes (PF and BCQI) in both performance parameters. In power saving mode, the performance of the PF, and BCQI degraded significantly

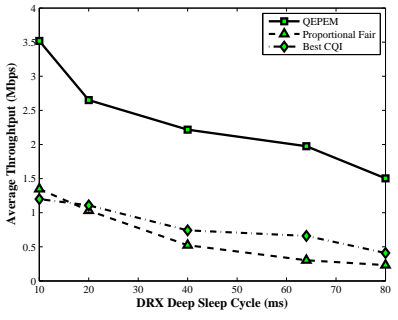

(a) Average Throughput

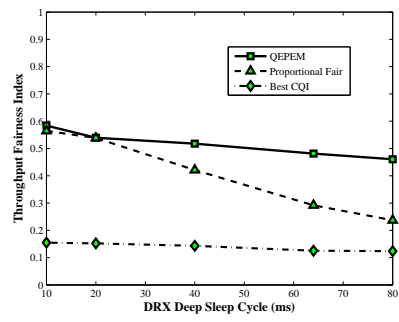

(b) Fairness Index
Figure 9: Vary Deep Sleep with Fixed Light Sleep=10 ms

in their respected order. The result clearly shows that the QEPEM is still performing better than the other schemes if the duration of the DRX Deep Sleep is increased. When the DRX Deep Sleep duration is increased continuously as shown in Figure 9, QEPEM has the highest performance index. PF had experienced poor system throughput as indicated by Figure 9a. Similarly, the performance of PF significantly degrades when the Deep Sleep duration exceed more than $20 \mathrm{~ms}$ as shown in Figure $9 \mathrm{~b}$.

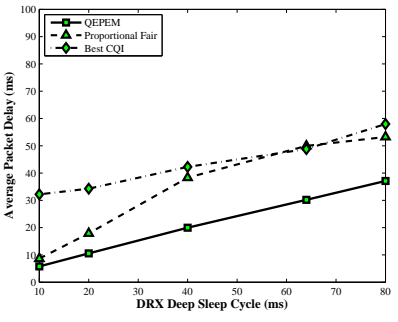

(a) Average Packet Delay

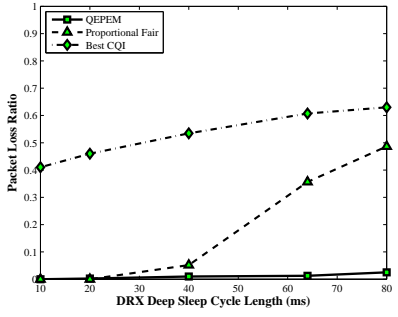

(b) Average Packet Loss Rate
Figure 10: Vary Deep Sleep with Fixed Light Sleep $=10 \mathrm{~ms}$

Figure 10 shows the performance of the three schedulers in terms of packet delay and loss rate. When the Deep Sleep duration increases, packets start to get delayed, as the packet delay is directly proportional to the power being saved through the DRX Deep and the Light Sleep duration. The simulation results clearly show that QEPEM method performs best, with less packet delay (see Figure 10a), and low packet loss rate (see Figure 10b) than other schedulers (PF and BCQI). The performance of $\mathrm{PF}$ is badly effected, as it has high packet loss rate when the duration of Deep Sleep increases from more than $40 \mathrm{~ms}$. BCQI has the worst performance in both packet delay and packet loss rate, due to its resource allocation policy.

\section{CONCLUSION}

In this paper, we have proposed a new QEPEM downlink scheduling algorithm for multimedia services, and it has been evaluated for delay sensitive multimedia VoIP traffic. The QEPEM method opts to enhance the QoE and provide better QoS by decreasing packet losses, improve fairness among UEs, and UEs meeting the QoS requirement of multimedia 
services. It has the capability to assure QoS in the power saving mode with high level of the users' satisfaction. The QEPEM method maximizes the user's QoE by using the user perception in its scheduling decision. The performance of QEPEM is compared with the traditional schemes through simulations. From the simulation results, it is observed that PLR has more influence on QoE as compared to delay. QEPEM method is evaluated in the power saving mode and the impact of the power saving on QoS and QoE is also examined. In the power saving mode, QEPEM method performance is remarkably better than the traditional schedulers with better user's experience because it allocates resources efficiently and fairly among the UEs. In future work, we shall compute how much power is saved by coordinating with a group of user devices with the DRX mechanism.

\section{REFERENCES}

[1] K. Aho, I. Repo, T. Nihtila, and T. Ristaniemi. Analysis of VoIP over HSDPA performance with discontinuous reception cycles. In Sixth International Conference on Information Technology: New Generations (ITNG), pages 1190 -1194, April 2009.

[2] Kian Chung Beh, S. Armour, and A. Doufexi. Joint time-frequency domain proportional fair scheduler with HARQ for 3GPP LTE systems. In IEEE 68th Vehicular Technology Conference(VTC Fall), pages 1 - 5, Sept. 2008.

[3] Huang Bo, Tian Hui, Chen Lan, and Zhu Jianchi. DRX-aware scheduling method for delay-sensitive traffic. IEEE Communications Letters, 14(12):1113 - 1115, Dec. 2010.

[4] Jung Yul Choi, Min-Gon Kim, Hongkyu Jeong, and Hong-Shik Park. Power-saving mechanisms for energy efficient IEEE $802.16 \mathrm{e} / \mathrm{m}$. Journal of Network and Computer Applications, Elsevier, 35(6):1728 - 1739, 2012.

[5] R. G. Cole and J. H. Rosenbluth. Voice over ip performance monitoring. SIGCOMM Computer Communication, 31(2):9-24, April. 2001.

[6] 3GPP; TSG RAN WG2 LTE Contribution. In Technical Specification, TS 36.300, N. R2-071285, 2007.

[7] O. Delgado and B. Jaumard. Joint admission control and resource allocation with GoS and QoS in LTE uplink. In IEEE GLOBECOM Workshops (GC Wkshps), pages 829 -833, Dec. 2010.

[8] Lijing Ding and R.A. Goubran. Speech quality prediction in voip using the extended e-model. In IEEE Global Telecommunications Conference,(GLOBECOM '03), volume 7, pages 3974-3978 vol.7, 2003.

[9] 3GPP; LTE RAN enhancements for diverse data applications. In $R A N$ Plenary Contribution, RP-110410, 2011.

[10] International Telecommunication Union (ITU-T); Perceptual evaluation of speech quality (PESQ): An objective method for end-to-end speech quality assessment of narrow-band telephone networks and speech codecs. In Recommendation P.862, Feb. 2001.

[11] Yong Fan, P. Lunden, M. Kuusela, and M. Valkama. Efficient semipersistent scheduling for VoIP on EUTRA downlink. In IEEE 68th Vehicular Technology Conference(VTC Fall), pages 1 - 5, Sept. 2008.

[12] International Telecommunication Union (ITU-T); Methodology for derivation of equipment impairment factors from subjective listeningonly tests. In Recommendation P.833, Nov. 2001.

[13] 3GPP; LTE Physical Layer Framework for Performance Verification Radio Access Network (RAN). In TSG-RAN1 no.48, R1-070674, 2007.

[14] International Telecommunication Union (ITU-T); Methods for subjective determination of transmissiom quality. In Recommendation P.800, 1996.

[15] S. Fowler. Study on power saving based on radio frame in LTE wireless communication system using DRX. IEEE Globecom Joint Workshop of SCPA and SaCoNAS, Dec. 2011.

[16] S. Fowler, R.S. Bhamber, and A. Mellouk. Analysis of adjustable and fixed DRX mechanism for power saving in LTE/LTE-Advanced. In IEEE International Conference on Communications (ICC), June 2012.

[17] Josep Colom Ikuno, Martin Wrulich, and Markus Rupp. System level simulation of LTE networks. In IEEE 71st Vehicular Technology Conference (VTC Spring), Taipei, Taiwan, May 2010.

[18] International Telecommunication Union (ITU-T); Transmission impairments due to speech processing. In Recommendation G.113, Nov. 2007.
[19] R. Jain. The art of computer systems performance analysis: techniques for experimental design, measurement, simulation and modeling. New York, John Wiley \& Sons, 1991.

[20] S.C. Jha, A.T. Ko, and R. Vannithamby. Optimization of discontinuous reception (drx) for mobile internet applications over LTE. In IEEE Vehicular Technology Conference (VTC Fall), Sept. 2012.

[21] Sunggeun Jin and D. Qiao. Numerical analysis of the power saving in 3GPP LTE Advanced wireless networks. IEEE Transactions on Vehicular Technology, 61(4):1779-1785, 2012.

[22] Yan Lin and Guangxin Yue. Channel-adapted and buffer-aware packet scheduling in LTE wireless communication system. In 4th International Conference on Wireless Communications, Networking and Mobile Computing (WiCOM), pages 1 - 4, Oct. 2008.

[23] Zhaoming Lu, Yan Yang, Xiangming Wen, Ying Ju, and Wei Zheng. A cross-layer resource allocation scheme for ICIC in LTE-Advanced. Journal of Network and Computer Applications, Elsevier, 34(6):1861 1868, 2011.

[24] International Telecommunication Union (ITU-T); The E model: a computational model for use in transmission planning. In Recommendation G.107, Dec. 2011.

[25] M.S. Mushtaq, A. Shahid, and S. Fowler. QoS-Aware LTE downlink scheduler for VoIP with power saving. In IEEE 15th International Conference on Computational Science and Engineering (CSE), Dec. 2012.

[26] International Telecommunication Union (ITU-T); Amendment 1: Defination of Quality of Experience. In Recommendation P.10/G.100, Jan. 2007.

[27] ETSI; Quality of Service (QoS) measurement methodologies. Annex e, method for determining an equipment impairment factor using passive monitoring. In TIPHONE TS101 329-5, 2002.

[28] 3GPP; Universal Mobile Telecommunications System (UMTS); User Equipment (UE) procedures in idle mode and procedures for cell reselection in connected mode. In Technical Specification, TS 25.304 version 5.9.0 Release 5, 2005.

[29] 3GPP; Medium Access Control(MAC) protocol specification. In Technical Specification, TS 36.321 version 10.2.0 Release 10, Mar. 2011.

[30] H.A.M. Ramli, R. Basukala, K. Sandrasegaran, and R. Patachaianand. Performance of well known packet scheduling algorithms in the downlink 3GPP LTE system. In IEEE 9th Malaysia International Conference on Communications (MICC), pages 815 - 820, Dec. 2009.

[31] 3GPP; Evolved Universal Terrestrial Radio Access (E-UTRA); Radio Resource Control (RRC). In Technical Specification, TS 36.331 version 11.0.0 Release 11, 2012.

[32] S. Sengupta, M. Chatterjee, S. Ganguly, and R. Izmailov. Improving RScore of VoIP Streams over WiMax. In IEEE International Conference on Communications, (ICC '06), volume 2, 2006.

[33] S. Uemura, N. Fukumoto, H. Yamada, and H. Nakamura. QoS/QoE measurement system implemented on cellular phone for NGN. In 5th IEEE Consumer Communications and Networking Conference (CCNC), pages $117-121$, Jan. 2008.

[34] Shun-Ren Yang and Yi-Bing Lin. Modeling UMTS discontinuous reception mechanism. IEEE Transactions on Wireless Communications, 4(1):312 - 319, Jan. 2005.

[35] Kaijie Zhou, Navid Nikaein, and Thrasyvoulos Spyropoulos. LTE/LTEA discontinuous reception modeling for machine type communications. IEEE Wireless Communications Letters, 2:102-105, 2013.

[36] Lei Zhou, Haibo Xu, Hui Tian, Youjun Gao, Lei Du, and Lan Chen. Performance analysis of power saving mechanism with adjustable DRX Cycles in 3GPP LTE. In IEEE 68th, Vehicular Technology Conference, (VTC Fall), pages 1-5, Sept. 2008. 